\title{
Arbitrary Conformal Transformations of Wave Functions
}

\author{
G. Ruffato $\odot,{ }^{1, *}$ E. Rotunno, ${ }^{2}$ L.M.C. Giberti, and V. Grillo ${ }^{2, \dagger}$ \\ ${ }^{1}$ Department of Physics and Astronomy 'G. Galilei', University of Padova, via Marzolo 8, 35125 Padova, Italy \\ ${ }^{2}$ CNR-Istituto Nanoscienze, Centro S3, Via G Campi 213/a, I-41125 Modena, Italy
}

(Received 21 March 2020; revised 23 February 2021; accepted 10 March 2021; published 13 May 2021)

\begin{abstract}
In this paper we prove that any conformal transformation of a wave can be produced via a suitably arranged cascade of two, or at most four, discrete phase elements satisfying Laplace's equation. Although this result is of general applicability, in the case of charged-matter waves it implies that such transformations can be exactly obtained by employing only electrostatic or magnetostatic phase elements. Furthermore, we illustrate how a basis for such generating phase elements is given by integer and fractional charge multipoles, proving that these transformations can be used to perform the efficient sorting of multipole-induced quantum states. This provides a fast, compact, and direct method to measure the strength and orientation of dipole systems and of astigmatism. It thus adds a further observable to the four whose spectrum can already be directly measured via spatial separation on the detector, i.e., position, momentum, energy, and orbital angular momentum. The results hold true in optics and for all kinds of charged-particle beams of sufficient coherence.
\end{abstract}

DOI: 10.1103/PhysRevApplied.15.054028

\section{INTRODUCTION}

In quantum mechanics, one of the advantages of the Heisenberg matrix formulation as opposed to the Schrödinger approach is that it provides a more general picture of the wave function, irrespective of the specific basis chosen for its representation [1,2]. Optics, on the other hand, is usually based on imaging and therefore on the space representation of the wave function. Indeed, the space representation still appears to have a privileged role in quantum measurement and the root of this seems to lie at the foundation of quantum mechanics itself [3]. For example, in optics one generally measures position (imaging), momentum (diffraction), and energy (spectroscopy) in terms of a spatial distribution of intensity, from which the desired quantities are calculated. The most recent addition to this limited set of quantities is the orbital angular momentum (OAM) [4-8], which can be measured via a quantum-state sorter, which produces the OAM decomposition of the wave function as a directly readable spatial modulation of intensity [9-11]. OAM decomposition is obtained via a suitable conformal transformation based

*gianluca.ruffato@unipd.it

†vincenzo.grillo@unimore.it

Published by the American Physical Society under the terms of the Creative Commons Attribution 4.0 International license. Further distribution of this work must maintain attribution to the author(s) and the published article's title, journal citation, and DOI. on log-pol mapping, effected by holograms [12-15] or refractive elements [10,16-18] calculated beforehand. If and how this strategy based on mapping could be pushed beyond the specific application to OAM was however never fully explored, until now.

Indeed, one can think of generalizing this "mapping" approach and thus obtain the wave-function decomposition over a basis of interest as a spatial modulation of intensity on the sensor plane, produced by a suitable unitary transformation. The most useful set of unitary transformations is provided by conformal transformations, of which the above-mentioned OAM decomposition is a special case.

In this paper we seek to express a general condition to which conformal transformations need to obey, with the aim to provide a general strategy to implement such a transformation experimentally. We find four main results, which - unless otherwise stated - apply to all types of wave functions:

(1) the sought condition is $\nabla^{2} \Omega=0$, where $\Omega$ is the phase imparted on the wave function; in the case of charged-matter waves, it means that arbitrary conformal transformations can be produced by phase elements consisting of a suitable arrangement of discrete electrostatic or magnetostatic sources; this implementation has two significant advantages compared to existing approaches: (i) it does not attenuate the beam and (ii) the resulting phase elements are tunable; 
(2) we prove that all conformal transformations of the wave function can be expressed as a combination of a subset of them, called circular-sector transformations;

(3) for circular-sector transformations we also provide a general recipe for designing the corresponding distribution of sources that is necessary to impart the required phase to charged-matter waves;

(4) a particularly interesting and promising consequence of all this is the possibility to effect an alternative decomposition on another basis of "multipole-phase" wave functions; this is significant since multipoles are of general interest in physics (magnetism, dielectrics, astigmatism, etc.).

\section{THEORY AND DISCUSSION}

In optics, the simplest setup for coordinate transformation consists of two phase elements [19]: the first one imparts the coordinate transformation, while the second one corrects for the phase distortion due to the ensuing free-space propagation. The wave function $\psi_{z}(u, v)$ at a distance $z$ from the first element is given by the Fresnel-Kirchhoff integral in the paraxial regime:

$$
\psi_{z}(u, v)=\frac{e^{i k z}}{i \lambda z} \int_{-\infty}^{+\infty} \int_{-\infty}^{+\infty} \psi_{0}(x, y) e^{i \Omega(x, y)} e^{-i k \frac{x u+y v}{z}} d x d y
$$

where $\psi_{0}(x, y)$ is the wave function impinging on the phase element, $k=2 \pi / \lambda$ is the wave-vector modulus, $(x, y)$ and $(u, v)$ are the Cartesian reference frames located at the input and output planes, respectively, and $\Omega(x, y)$ is the phase imparted by the element. Applying the stationary-phase approximation [20] to the diffraction integral Eq. (1) yields $\psi_{z}(u, v) \approx$ $\sum_{\left\{\left(x^{*}, y^{*}\right)\right\}} \psi_{0}\left(x^{*}, y^{*}\right) e^{i \Phi\left(x^{*}, y^{*}\right)} k \sigma / \sqrt{|H|}$, where $\left\{\left(x^{*}, y^{*}\right)\right\}$ are the saddle points of the total phase function $\Phi \equiv \Omega-$ $k(x u+y v) / z, H$ is the Hessian determinant of $\Phi$, and $\sigma=\operatorname{sgn}\left(\partial^{2} \Phi / \partial x^{2}\right)$ when $H>0, \sigma=-i$ otherwise. The stationary condition $\nabla \Phi=0$ leads to the following relation between the two-dimensional (2D) gradient of the phase pattern $\Omega(x, y)$ and the point $\rho \equiv[u(x, y), v(x, y)]$ :

$$
\rho=\frac{z}{k} \nabla \Omega .
$$

Therefore, the phase element can be thought of as realizing a mapping between a point $(x, y)$ on the input plane, and a point $[u(x, y), v(x, y)]$ on the destination plane. As a consequence of Eq. (2), $\boldsymbol{\rho}$ is irrotational, i.e., $\nabla \times \boldsymbol{\rho}=\mathbf{0}$ [21], and in particular

$$
\frac{\partial u}{\partial y}=\frac{\partial v}{\partial x}
$$

An especially interesting case occurs when the complex version of the above transformation $\rho_{c}=u(x, y)+i v(x, y)$ defines a conformal mapping, i.e., it is holomorphic or antiholomorphic. Then Eq. (3) implies that $\rho_{c}$ must necessarily be antiholomorphic, i.e., $\partial_{\zeta} \rho_{c}=1 / 2\left(\partial_{x}-i \partial_{y}\right) \rho_{c}=$ 0 , where $\zeta=x+i y$, and

$$
\frac{\partial u}{\partial x}=-\frac{\partial v}{\partial y} .
$$

Taking the divergence of Eq. (2) and substituting Eq. (4) yields

$$
\nabla^{2} \Omega=0
$$

Therefore, the phase function of an optical element performing an antiholomorphic (conformal) transformation must be harmonic, i.e., a solution of Laplace's equation in 2D. The same condition Eq. (5) holds for the second element, which can be proved using the same mathematical argument, or simply a time-reversal argument $[11,12]$.

Due to the work by Hossack et al. [19], we refer to this kind of two-element antiholomorphic (conformal) mappings as Hossack's transformations.

The general statement at the core of this paper is that every Hossack transformation can be effected by a cascade of suitable harmonic phase elements. Because of Maxwell's equations, in the electron-optics scenario such a transformation inherently admits an electrostatic implementation: in fact, the phase imparted to the electron wave function is proportional to the integral of the potential along the propagation direction, which satisfies Laplace's equation $\nabla^{2} \phi=0$ everywhere except on the electrodes [22]: since the same condition holds for the integrated potential, the desired transformation can be reproduced by means of a finite combination of electrodes (or currents), as stated in claim 1 in the introduction. The claim can be extended also to holomorphic mappings by cascading two appropriate Hossack's transformations (four elements in total, see Appendix E for details). Then, since holomorphic and antiholomorphic transformations cover the whole space, we prove the general validity of claim 1 for any conformal mapping.

The log-pol transformation, which is the basis for the OAM sorter, satisfies the above condition Eq. (5). Indeed the OAM sorter can be built with charged needles [16-18]. Besides the log-pol case, it is interesting to look at a family of polar mappings that has been recently introduced in optics to perform circular-sector transformations (CSTs), the combination of which permits the OAM quantum number of vortex beams to be multiplied or divided [23,24]. Solving the 2D Laplace equation in polar coordinates $(r, \vartheta)$ by separation of variables yields

$$
\Omega(r, \vartheta)=A r^{m} \cos \left(m \vartheta+\vartheta_{0}\right),
$$



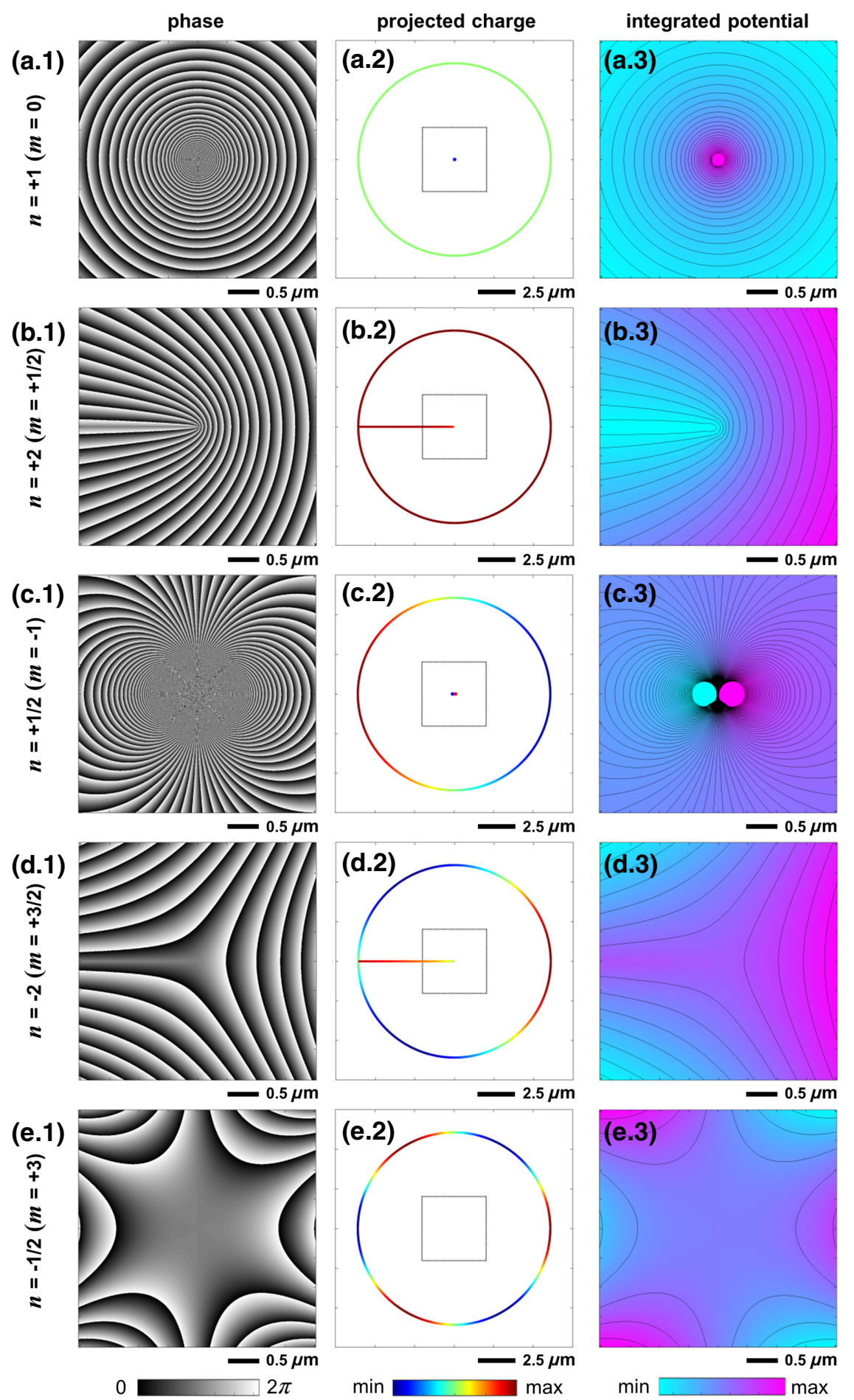
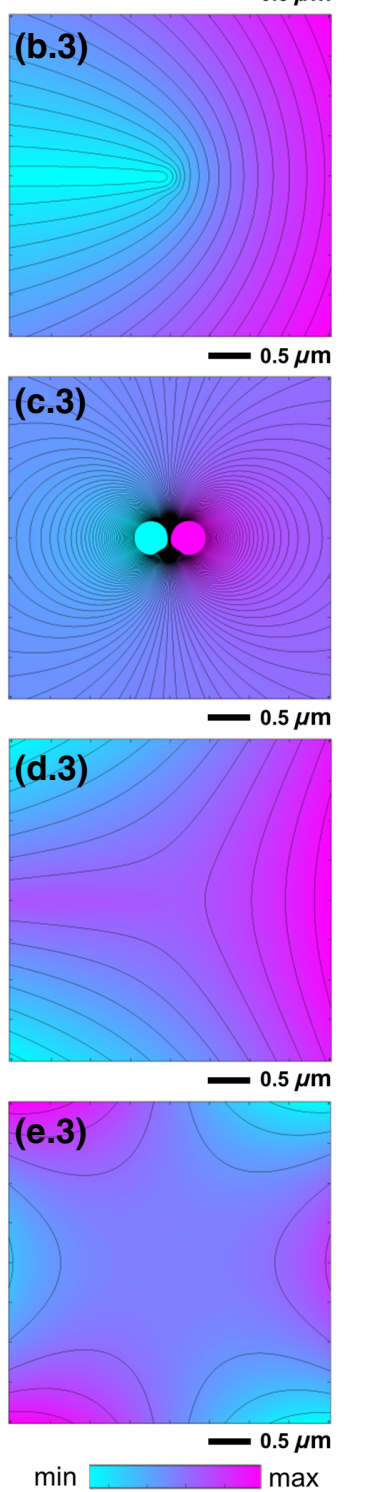

FIG. 1. n-fold circular-sector transformation elements for different values of $n$ : $n=+1$ (a), $n=+2$ (b), $n=+1 / 2$ (c), $n=-2$ (d), $n=-1 / 2$ (e). The corresponding phase plates (first column) exhibit a multipole phase pattern of order $m=1-1 / n$. Projected charge distribution (second column) and corresponding integrated potential (third column). Design parameters: $a=4.0 \mu \mathrm{m}$, $b=2.5 \mu \mathrm{m}, f=50 \mathrm{~cm}, \lambda=1.9687 \mathrm{pm}(300$ $\mathrm{KeV})$. All the integrated potentials refer to the black dashed boxes in the projected charge graphs. where $m$ is an integer. Applying Eq. (2) yields the corresponding conformal transformation:

$$
(\rho, \varphi)=\left[z A m r^{m-1} / k,(1-m) \vartheta-\vartheta_{0}\right],
$$

i.e., the functional relation between the two reference frames $(r, \vartheta)$ and $(\rho, \varphi)$ on the input and output planes, at distance $z$, respectively. By substituting $m=1-1 / n$, Eq. (6) provides exactly the functional dependence of the phase element performing a conformal CST by a factor $n$, as described by Eq. (7), that is $(\rho, \varphi)=$ $\left(z A m r^{-1 / n} / k, \vartheta / n-\vartheta_{0}\right)$ (see Appendix A). The corresponding phase corrector is the element performing the inverse transformation, i.e., mapping a circular sector with angular amplitude $2 \pi / n$ onto the whole circle. Its phase function is provided by the substitution $n \rightarrow 1 / n$ and is described by the phase profile Eq. (6), with $m^{\prime}=$ $1-n=m /(m-1)$. The trivial CST given by $n=+1$ is 

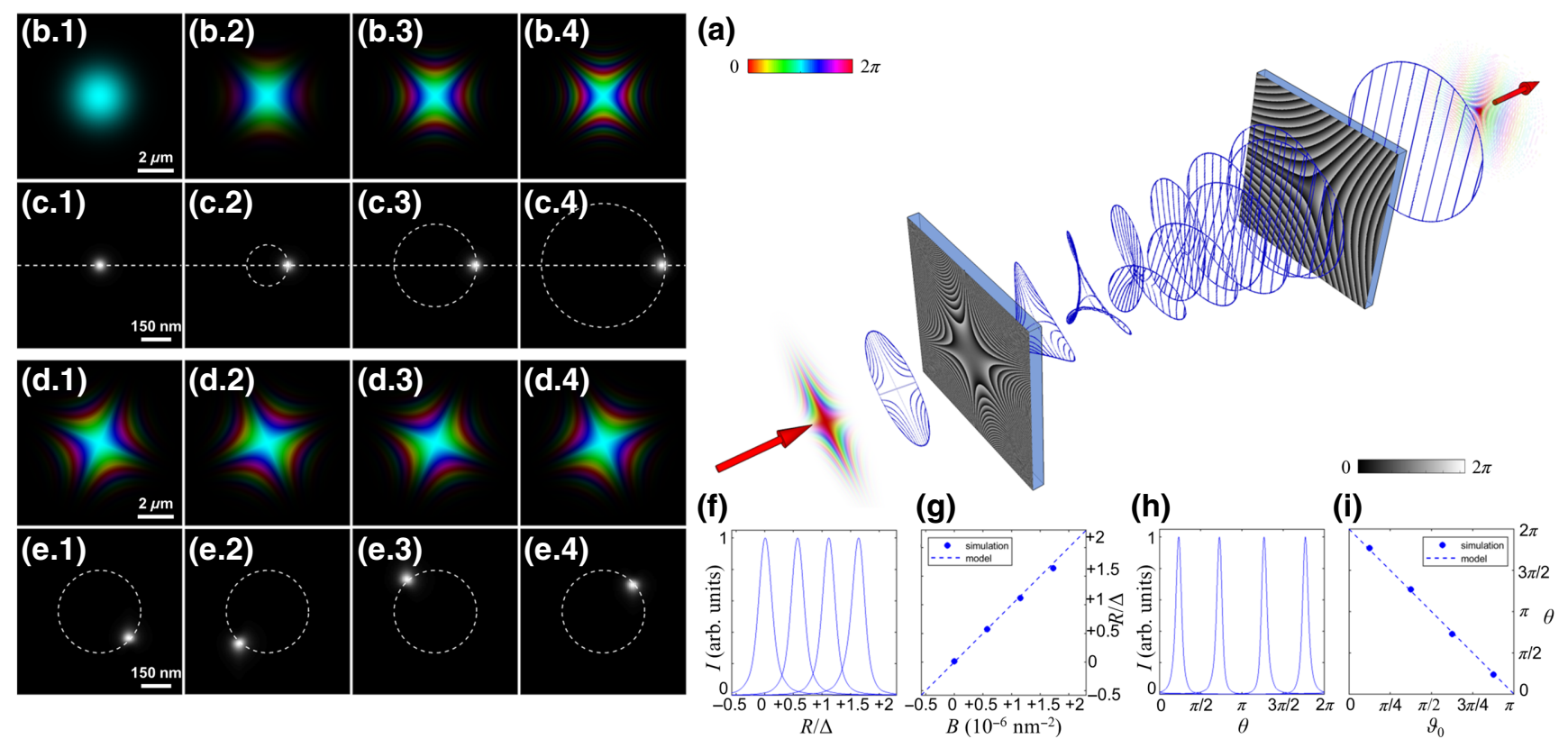

FIG. 2. Twofold astigmatism measurement using circular-sector transformation with $n=-1 / 2$. (a) The input phase is transformed conformally into a linear phase gradient, determining a bright spot in far field with position depending on the astigmatism strength $B$ and in-plane rotation angle $\vartheta_{0}$. (b) Numerical simulations for increasing values of $B$ corresponding to 0 (b.1), 8 (b.2), 16 (b.3), and $24 \mu \mathrm{m}$ (b.4) of astigmatism for a focal length of $3.33 \mathrm{~mm}$ (realistic value for a transmission electron microscope), fixed $\vartheta_{0}=0$. The generated spot in far field (c.1-4) shifts proportionally to $B$. (f) Cross section and (g) far-field position $R$ (normalized by $\Delta=f_{\mathrm{L}} / \mathrm{k} b^{2} / a$ ) as a function of $B$. (d) Simulations for increasing rotation angle, fixed astigmatism of $16 \mu \mathrm{m}$. The far-field spot (e.1-4) appears at an angular position $\theta$ equal to $-2 \vartheta_{0}$. (h) Cross section and (i) angular position as a function of the input rotation. Transformation parameters $a=4.0 \mu \mathrm{m}, b=2.5 \mu \mathrm{m}, f=50 \mathrm{~cm}, \lambda=1.9687 \mathrm{pm}(300 \mathrm{KeV}), f_{\mathrm{L}}=1.2 \mathrm{~m}$. In (a), (b.1-4), and (d.1-4), brightness and colors refer to intensity and phase, respectively.

effected by the axially symmetric phase element $\Omega(r, \vartheta)=$ $A \log (r / b), b$ being a design parameter. Again, this is a solution of Eq. (5), which completes the set provided by Eq. (6). It is worth noting that an integer $n$ corresponds to a fractional value of $m$. In this case Eq. (6) is still a solution of Eq. (5) save for the points $\vartheta=\pi$, where the phase has a discontinuity in its derivatives.

Finally, by using the uniqueness of the analytical continuation on a circular domain, it is easy to prove (see Appendix A) that any harmonic function-i.e., any function satisfying Eq. (5) - can be expressed as a sum of the separable harmonic functions given by Eq. (6), which proves claim 2.

In Fig. 1, the phase patterns of $n$-fold CSTs are shown for different values of the parameter $n$ : for positive $n$, the visual analogy between the phase correctors and the projected potentials of electric multipoles is already quite evident. However, this is more than an analogy, since it can be proved (see Appendix B) that the phase in Eq. (6) is proportional to the integral over $z$ of the potential $V(\mathbf{s}) \propto s^{m-1} P_{|m|}^{|m|}(\cos \gamma) \cos (m \vartheta)$ of an in-plane electric $2|m|$ pole, where $\mathbf{s}=(\mathbf{r}, z), \gamma$ is the angle between $\boldsymbol{s}$ and the $z$ axis, $P_{|m|}^{|m|}(\cdot)$ is the associated Legendre polynomial with both indices equal to $|m|$. For charged-matter waves, e.g., electrons, this result provides a general method to impart CST phase patterns by using electrostatic elements featuring the corresponding in-plane electric charge distributions. For instance, Figs. 1(a) and 1(c) show patterns corresponding to a single charge and a dipole, implementing the phase correctors of CSTs with $n=+1$ and $n=+2$, respectively. While for the trivial case, the $n=+1$ transformer and phase corrector coincide, for $n=+2$ the phase pattern of the transformer [Fig. 1(b)] exhibits a discontinuity at $\vartheta=\pi$. The same discontinuity is found at negative integer values, as shown in Fig. 1(d) for $n=-2$. By solving the corresponding Poisson equation, one can prove (see Appendix C) that the phase pattern for $n \in$ $\{\mathbb{Z} \backslash\{0, \pm 1\}\}$ has the same form as the integrated electrostatic potential generated by a linear distribution of charge, $\rho_{L}(r)=Q_{L} r^{-1 / n} \delta(\vartheta-\pi)$, where $Q_{L}=-2 \varepsilon_{0} A m \sin (m \pi)$. The corresponding field is that of a fractional-order multipole, as defined in Refs. [25,26]. An appropriate boundary condition can be added at a radius $R$, defined by a charge density $\rho_{R}(\vartheta)=Q_{R} \cos (m \vartheta) \delta(R-r)$, where $Q_{R}=$ $2 \varepsilon_{0} A m R^{m-1}$ (Appendix C) in order to ensure the chargeneutrality condition and the convergence of the integral [27].

Analogously, for negative fractional values of $n$, the phase-corrector patterns resemble the integrated field of magnetic multipoles [see Fig. 1(e) for $n=-1 / 2$ ], and the 

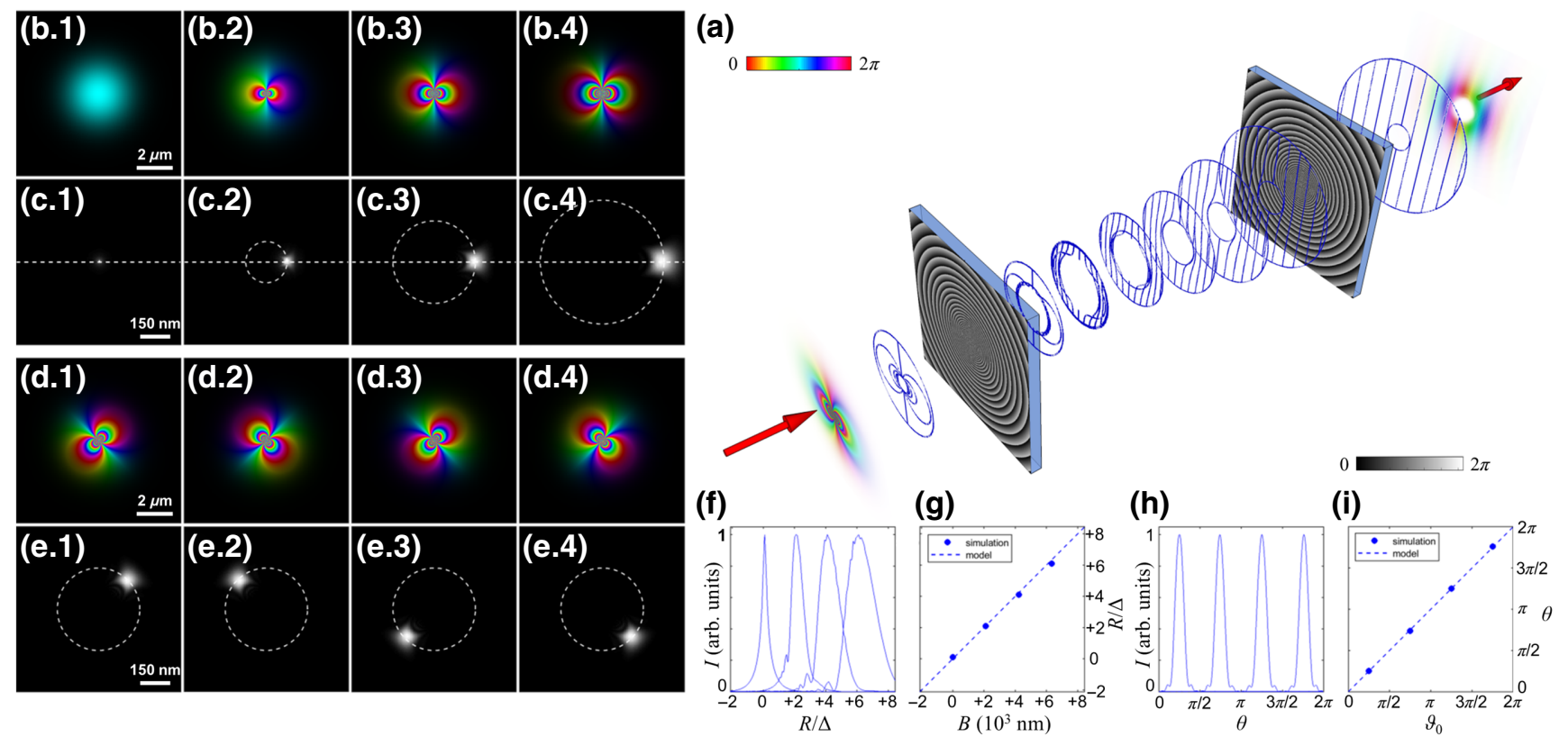

FIG. 3. (a) Magnetic dipole measurement using CST with factor $n=+1$. (b) Simulations for increasing strength $B$ corresponding to 0 (b.1), 6 (b.2) 12 (b.3), and $18 \times 10^{6} \mu_{B}$ (b.4), and fixed $\vartheta_{0}=0$, and corresponding far field (c.1-4). The value of $10 \times 10^{6} \mu_{B}$ corresponds to typical magnetic nanoparticles of around 50-nm diameter (see Ref. [35]). (f) Cross section and (g) far-field position (normalized by $\left.\Delta=f_{\mathrm{L}} / k b^{-1} / a\right)$ as a function of $B$. (d) Simulations for increasing $\vartheta_{0}$, fixed $B=12 \times 10^{6} \mu_{B}$. The far-field spot (e.1-4) appears at an angular position $\theta$ equal to $\vartheta_{0}$. (h) Cross section and (i) angular position as a function of $\vartheta_{0}$. Same transformation parameters as in Fig. 2. In (a), (b.1-4), and (d.1-4), brightness and colors refer to intensity and phase, respectively.

corresponding integrated potentials [Fig. 1(e.3)] can be generated by the ring of multipolar charge distribution with the appropriate $m$ integer value [Fig. 1(e.2)], without any linear charge distribution.

The charge distributions for all relevant CST phase elements are summarized in Fig. 1. The desired charge distribution can be realized by shaping the electrodes appropriately (by solving the inverse problem $[28,29]$ ) or by substituting the required charges with vertical currents [30]. This proves claim 3.

In practice, this method provides a lossless approach to the beam shaping of charged-matter waves, analogous to phase manipulation in optics with refractive and diffractive elements (including spatial light modulators) [31] and metasurfaces [32]. It also provides an alternative recipe for conformal beam shaping in optics. We foresee two significant domains of application in electron optics. On the one hand, we can produce many different wavefronts by just finding the appropriate conformal transformation. This is a valid alternative to hole-based programmable phase plates [33], offering the added advantage that there is no obstruction to the beam. On the other hand, we can extend the paradigm of quantitative measurement by designing transformations that map certain observables of interest into specific points in the far field, on the detector plane.

For instance, in the case of the log-pol optical transformation, the corresponding family of wave functions is represented by beams with an azimuthal phase gradient (vortex beams) and the composition of logpol mapping and subsequent Fourier transform has been widely demonstrated to work as an OAM sorter [9].

In the case of CSTs, the corresponding family is comprised of wave functions endowed with "multipole-phase" profiles:

$$
\psi(r, \vartheta)=\exp (i k z) \exp \left\{i B r^{m} \cos \left[m\left(\vartheta-\vartheta_{0}\right)\right]\right\} .
$$

That is, integrating the electric and magnetic potential along $z$ shows that this is the phase of a charged particle that has interacted with an in-plane electric (or magnetic) multipole of order $m$, where $B$ is related to the multipole moment (see Appendix D). If we consider the input wave function Eq. (8) and apply the CST with a factor $n=-1 / m$, the following output is obtained:

$$
\psi_{z}(\rho, \varphi) \propto C \exp \left[i B^{\prime} \rho \cos \left(\varphi+m \vartheta_{0}\right)\right] .
$$

The output wave function is endowed with a linear phase term, with spatial frequency $B^{\prime}=B b^{m} / a,(a, b)$ being the scaling parameter of the applied transformation (see Appendix D). The far field at a distance $d$ will exhibit a spot forming an angle $-m \vartheta_{0}$ with the $x$-axis positive direction, and shifted by an amount $d B^{\prime} / k$ with respect to the origin. This proves claim 4. For instance, an $n=-1 / 2$ CST followed by a Fourier transform transforms an external quadrupole $(m=+2)$ phase object into 
a spot, as shown in Fig. 2, allowing one to measure the quadrupole moment and its orientation $\vartheta_{0}$ by reading them directly as a point signal from the sensor. This method to measure astigmatism could even be combined with a Zemlin scheme for fast measurement of higher-order aberrations [34]. Even more interesting is the fact that we can measure the strength of a dipole moment, a fundamental quantity in material science [22]: for $m=-1$, we can transform the dipole phase profile into a linear one, thanks to the trivial CST defined by $n=+1$. Figure 3 shows the transformation of an input dipole phase profile into an output spot for different values of dipole moment and in-plane orientation: the axial displacement of the far-field spot increases proportionally to the dipole strength, while its angular position is equal to the rotation angle. This mapping to a point, which is limited and approximate in the log-pol case [15], is instead exact here. The linear dependence of the displacement on the dipole moment indicates that, in principle, one could measure both the strength and orientation of very small dipoles, using a method that is much faster than holography and therefore less affected by drift. This approach also lends itself to studies of magnetic dipole evolution at very short time scales.

We thus prove the deep link between Hossack's transformations and the landscape of harmonic phases, which for charged particles allows the use of simple electrodes to produce arbitrary conformal mappings of wave functions. In electron microscopy this provides a general scheme for beam shaping and for the optimal measurement of quantum observables. In particular, we prove that circularsector transformations can transform multipole-induced phases into a spot whose position is dependent on the strength and orientation of the multipole momentum, thus showing it is possible to directly measure astigmatism and dipole moments with a compact setup.

\section{ACKNOWLEDGEMENTS}

V.G. thanks Prof. Giulio Pozzi for fruitful discussions. This work is supported by Q-SORT, a project funded by the European Union's Horizon 2020 Research and Innovation Program under Grant Agreement No. 766970, and by VORTEX3, a project funded by the Italian Ministry of Defence.

\section{APPENDIX A: CIRCULAR-SECTOR TRANSFORMATIONS AS GENERAL SOLUTIONS OF LAPLACE'S EQUATION IN 2D}

In this section, we prove how the general solution of the 2D Laplace equation in polar coordinates is endowed with a potential profile inducing a circular-sector transformation. As a matter of fact, a general solution of Laplace's equation $\nabla^{2} \Omega=0$ in polar coordinates $(r, \vartheta)$, under variable separation, is given by

$$
\Omega(r, \vartheta)=A r^{m} \cos \left(m \vartheta+\vartheta_{0}\right) .
$$

The solution of the Fresnel-Kirchhoff integral in the stationary-phase approximation expresses a mapping between the wave function on the input plane $(r, \vartheta)$ and the transformed wave function on the output plane $(\rho, \varphi)$ upon propagation after a phase element described by Eq. (A1) [19]. This mapping is related to the gradient of the phase $\Omega$ as it follows

$$
\rho=\frac{f}{k} \nabla \Omega,
$$

where $\rho=\rho(\cos \varphi, \sin \varphi)$. The last relation allows one to obtain the conformal transformation induced by the given phase element, i.e., the functional relation between the two polar reference frames $(r, \vartheta)$ and $(\rho, \varphi)$ on the input and output planes, respectively. From Eq. (A1) it follows

$$
\left\{\begin{array}{l}
\frac{\partial \Omega}{\partial x}=A m r^{m-1} \cos \left(\vartheta-m \vartheta-\vartheta_{0}\right) \\
\frac{\partial \Omega}{\partial y}=A m r^{m-1} \sin \left(\vartheta-m \vartheta-\vartheta_{0}\right)
\end{array} .\right.
$$

After inserting the previous derivatives in Eq. (A2) it is straightforward to obtain

$$
(\rho, \varphi)=\left[\frac{f}{k} A m r^{m-1},(1-m) \vartheta-\vartheta_{0}\right] .
$$

Under the substitution $m=1-1 / n$, Eq. (A4) describes a conformal circular-sector transformation by a factor $n$, mapping the whole circle onto a $2 \pi / n$ circular sector $[23,24]$ :

$$
(\rho, \varphi)=\left[a(r / b)^{-1 / n}, \vartheta / n-\vartheta_{0}\right],
$$

where $a$ and $b$ are scaling parameters. With the previous definitions, Eq. (A1) can be rewritten in the form:

$$
\Omega(r, \vartheta)=\frac{k a b}{f}\left(\frac{r}{b}\right)^{1-(1 / n)} \frac{\cos \left\{[1-(1 / n)] \vartheta-\vartheta_{0}\right\}}{1-(1 / n)} .
$$

The corresponding phase corrector is the optical element performing the inverse optical transformation, i.e., mapping a circular sector with angular amplitude $2 \pi / n$ onto the whole circle. Its phase function is provided by a circularsector transformer [Eq. (A6)] under the substitution $n \rightarrow$ $1 / n, a \leftrightarrow b$, that is

$$
\Omega_{\mathrm{PC}}(\rho, \varphi)=\frac{k a b}{f}\left(\frac{\rho}{a}\right)^{1-n} \frac{\cos \left[(1-n) \varphi+n \vartheta_{0}\right]}{1-n} .
$$

Analogously, it can be shown that the trivial circular-sector transformation given by $n=+1$ corresponds to the logarithmic radial solution of the $2 \mathrm{D}$ Laplace equation, that 
is

$$
\Omega(r, \vartheta)=A \log \left(\frac{r}{b}\right)
$$

Its partial derivatives are given by

$$
\left\{\begin{array}{l}
\frac{\partial \Omega}{\partial x}=\frac{A}{r} \cos (\vartheta) \\
\frac{\partial \Omega}{\partial y}=\frac{A}{r} \sin (\vartheta)
\end{array} .\right.
$$

As done above, after inserting the previous expressions into Eq. (A2) it is straightforward to obtain the relations between the two reference frames:

$$
(\rho, \varphi)=\left(\frac{f}{k} \frac{A}{r}, \vartheta\right)
$$

which describes a conformal circular-sector transformation [Eq. (A5)] with $n=+1$ (i.e., $m=0$ ) and the definition $A=$ $k a b / f$.

In the following, we provide a straightforward proof that any harmonic function can be developed as a series of general solutions as the one in Eq. (A1). For a given harmonic function $F(r, \vartheta)$ satisfying Laplace's equation in $2 \mathrm{D}$, i.e., $\nabla^{2} F=0$, we consider a circle $r=R$. Then $F$ can be developed in cosine series according to

$$
F(r=R, \vartheta)=\sum_{m} a_{m} \cos (m \vartheta) .
$$

By taking $a_{m}=b_{m} R^{m}$, this is exactly the value that the function

$$
G(r, \vartheta)=\sum_{m} b_{m} r^{m} \cos (m \vartheta)
$$

would take at the same points. For the theorem of uniqueness of the analytical continuation, the function is therefore the same over the full domain.

\section{APPENDIX B: PROJECTED POTENTIAL PRODUCED BY A MULTIPOLE FIELD}

In this section we demonstrate that the projected potential generated by an electric multipole of order $2^{l}$ exhibits the same spatial dependence as in Eq. (A1), with negative $m=-l$, therefore the transferred phase term induces a $n$ fold circular-sector transformation, with $n=1 /(1-m)$, as shown above.

The Coulomb potential of a continuous charge distribution $\rho(\mathbf{s})$ is given by

$$
\varphi(\mathbf{R})=\frac{1}{4 \pi \varepsilon_{0}} \int d V \frac{\rho(\mathbf{s})}{|\mathbf{R}-\mathbf{s}|} .
$$

Since we want to know the potential far away from said charge distribution, then we can expand the denominator in the Coulomb potential according to [36]

$$
\frac{1}{|\mathbf{R}-\mathbf{s}|}=\sum_{l=0}^{+\infty} \frac{s^{l}}{R^{l+1}} P_{l}(\cos \gamma),
$$

where $\gamma$ is the angle formed by the vectors $\mathbf{R}$ and $\mathbf{s}$. Then the Coulomb potential can be expressed as

$$
\varphi(\mathbf{R})=\sum_{l=0}^{+\infty} \frac{1}{4 \pi \varepsilon_{0} R^{l+1}} \int d V \rho(\mathbf{s}) s^{l} P_{l}(\cos \gamma)
$$

Consequently, we can decompose the potential into a series of multipole potentials:

$$
\varphi(\mathbf{R})=\sum_{l=0}^{+\infty} \frac{M_{l}(\mathbf{R})}{4 \pi \varepsilon_{0} R^{l+1}},
$$

where $M_{l}(\mathbf{R})$ is the $2^{l}$-pole moment of the charge distribution in the direction of the vector $\mathbf{R}$. It is useful to consider the expansion of the Legendre polynomial $P_{l}(\cos \gamma)$ into spherical harmonics $Y_{l}^{m}(\vartheta, \varphi)=P_{l}^{m}(\cos \vartheta) e^{i m \varphi}\left(P_{l}^{m}\right.$ being the associate Legendre polynomial), that is

$$
P_{l}(\cos \gamma)=\frac{4 \pi}{2 l+1} \sum_{m=-l}^{+l} Y_{l}^{m}(\vartheta, \varphi) Y_{l}^{m}(\theta, \phi)^{*}
$$

where $(\vartheta, \varphi)$ and $(\theta, \phi)$ are the angles of vectors $\mathbf{R}$ and $\mathbf{s}$ in spherical coordinates, respectively. Substituting the last expansion into Eq. (B3), we obtain the following expression for Eq. (B4):

$$
\varphi(\mathbf{R})=\frac{1}{4 \pi \varepsilon_{0}} \sum_{l=0}^{+\infty} \sum_{m=-l}^{+l} M_{l}^{m} \frac{Y_{l}^{m}(\vartheta, \varphi)}{R^{l+1}},
$$

where the spherical harmonics of multipoles are given by

$$
M_{l}^{m}=\frac{4 \pi}{2 l+1} \int d V s^{l} Y_{l}^{m}(\theta, \phi)^{*} \rho(\mathbf{s}) .
$$

If we limit our analysis to an in-plane multipole, then the corresponding $2^{l}$-pole contribution can be expressed as

$$
\varphi_{2 l}(R, \vartheta, \varphi)=\frac{1}{4 \pi \varepsilon_{0}} M_{l}^{l} \frac{P_{l}^{l}(\cos \vartheta)}{R^{l+1}} \cos (l \varphi)
$$


The corresponding projected potential, as a result of the integration in $z=R \cos \vartheta$, in given by

$$
\begin{aligned}
\varphi_{2 l}^{z}(\rho, \varphi) & =\frac{1}{4 \pi \varepsilon_{0}} \int_{-\infty}^{+\infty} d z M_{l}^{l} \frac{P_{l}^{l}(\cos \vartheta)}{R^{l+1}} \cos (l \varphi) \\
& =-\frac{1}{4 \pi \varepsilon_{0}} \cos (l \varphi) \int_{0}^{\pi} d \vartheta M_{l}^{l} \frac{P_{l}^{l}(\cos \vartheta)}{R^{l}} \sin \vartheta \\
& =-\frac{1}{4 \pi \varepsilon_{0}} \frac{\cos (l \varphi)}{\rho^{l}} \int_{0}^{\pi} d \vartheta M_{l}^{l} \frac{P_{l}^{l}(\cos \vartheta)}{\left(1+\cos ^{2} \vartheta\right)^{l / 2}} \sin \vartheta \\
& =\frac{1}{4 \pi \varepsilon_{0}} \frac{\cos (l \varphi)}{\rho^{l}} C_{l}^{l},
\end{aligned}
$$

where we introduce the polar coordinates $(\rho, \varphi)$ on the plane $z=0$, and we define $C_{l}^{l}=-\int_{0}^{\pi} d \vartheta M_{l}^{l} P_{l}^{l}(\cos \vartheta) \sin \vartheta /$ $\left(1+\cos ^{2} \vartheta\right)^{l / 2}$.

\section{APPENDIX C: CHARGE-DISTRIBUTION CALCULATION}

In this section, we perform the analytical calculation of the charge distribution required for the desired circularsector transformation when $m>1$, i.e., $n<0$, and in the case $0<m<1$, i.e., integer positive $n$. For fractional positive $n$, i.e., integer $m \leq 0$, the phase is proportional to the projected potential of an electric multipole generated by the proper distribution of charges around the origin, i.e., electric monopole, electric dipole $(n=+1 / 2, m=-1)$, electric quadrupole $(n=+1 / 3, m=-2)$, etc.), as shown in the previous section.

The calculation of the charge density is based on the relationship between the two-dimensional phase shift and the projected charge. A quick approach is to take the Laplacian of the phase element, which corresponds to the projected charge density:

$$
\nabla^{2} \phi(r, \vartheta)=-\frac{\rho(r, \vartheta)}{\varepsilon_{0}} .
$$

In polar coordinates we have

$$
\left[\frac{1}{r} \frac{\partial}{\partial r}\left(r \frac{\partial}{\partial r}\right)+\frac{1}{r^{2}} \frac{\partial^{2}}{\partial \vartheta^{2}}\right] \phi(r, \vartheta)=-\frac{\rho(r, \vartheta)}{\varepsilon_{0}} .
$$

We insert in the phase function in the previous equation:

$$
\phi(r, \vartheta)=A r^{m} \cos (m \vartheta) \Theta(R-r), \quad \vartheta \in(-\pi, \pi],
$$

where $\Theta(\cdot)$ is the Heaviside function: $\Theta(x)=1$ when $x \geq 0, \Theta(x)=0$ otherwise. When $m$ is fractional, a discontinuity line appears at $\vartheta=\pi$ for the first derivative in the azimuthal direction. The two contributions on the left side of Eq. (C2) can be easily calculated as it follows

$$
\begin{aligned}
& \frac{1}{r} \frac{\partial}{\partial r}\left(r \frac{\partial}{\partial r}\right) \phi(r, \vartheta)=A m^{2} r^{m-2} \cos (m \vartheta) \Theta(R-r) \\
& -2 A m R^{m-1} \cos (m \vartheta) \delta(R-r), \\
& \frac{1}{r^{2}} \frac{\partial^{2}}{\partial \vartheta^{2}} \phi(r, \vartheta)=-A m^{2} r^{m-2} \cos (m \vartheta) \Theta(R-r) \\
& \quad+2 A m r^{m-2} \sin (m \pi) \delta(\vartheta-\pi) \Theta(R-r),
\end{aligned}
$$

where $\delta(\cdot)$ is the Dirac $\delta$ function. Therefore, the total projected charge density $\rho$ can be split into two contributions, a ring charge distribution $\rho_{R}$ and a line charge distribution $\rho_{L}$, given by

$$
\rho_{R}(\vartheta)=Q_{R} \cos (m \vartheta) \delta(R-r)
$$

$$
\rho_{L}(r)=Q_{L} r^{m-1} \Theta(R-r) \delta(\vartheta-\pi),
$$

where $Q_{R}=2 \varepsilon_{0} A m R^{m-1}, Q_{L}=-2 \varepsilon_{0} A m \sin (m \pi)$. The radius $R$ of the charged ring can be selected in order to satisfy the total charge neutrality.

For positive integer $m$, the phase resembles the one induced by an external magnetic multipole, for which only the ring distribution is required, since there is no discontinuity in the azimuthal direction. On the other hand, for negative integer $m$, the phase is generated by a proper distribution of electric poles around the origin, as mentioned above.

For a given charge density, the generated potential is calculated by applying the integral

$$
\phi(\mathbf{r}, z)=\frac{1}{4 \pi \varepsilon_{0}} \iint \frac{\rho\left(\mathbf{r}^{\prime}\right)}{\sqrt{\left|\mathbf{r}-\mathbf{r}^{\prime}\right|^{2}+z^{2}}} r^{\prime} d r^{\prime} d \vartheta^{\prime} .
$$

Finally, the integrated potential is given by [22]

$$
\phi^{z}(r, \vartheta)=\int_{-\infty}^{+\infty} \phi(\mathbf{r}, z) d z
$$

\section{APPENDIX D: SORTING OF MULTIPOLE PHASE PROFILES WITH A CIRCULAR-SECTOR TRANSFORMATION}

We consider a wave function with the input phase

$$
\Phi^{i n}(r, \vartheta)=B r^{m} \cos \left[m\left(\vartheta-\vartheta_{0}\right)\right] .
$$


After applying a circular-sector transformation of a factor $n$, and parameters $(a, b)$, that is

$$
(\rho, \varphi)=\left[a\left(\frac{r}{b}\right)^{-(1 / n)}, \frac{\vartheta}{n}\right]
$$

The output phase is given by

$$
\Phi^{\text {out }}(\rho, \varphi)=B^{\text {out }} \rho^{-m n} \cos \left(m n \varphi-m \vartheta_{0}\right),
$$

where $B^{\text {out }}=B b^{m} / a^{-m n}$. With the choice $n=-1 / m$, Eq. (D3) reduces to the plane-wave term:

$$
\Phi^{\text {out }}(\rho, \varphi)=B^{\text {out }} \rho \cos \left(\varphi+m \vartheta_{0}\right) .
$$

The far field at a distance $z$ exhibits a spot forming an angle $-m \vartheta_{0}$ with the $x$ axis and shifting $z B^{\text {out }} / k$ with respect to the optical axis. In the main text, we consider the following examples.

Input external quadrupole field (astigmatism): $\boldsymbol{m}=\mathbf{+ 2}$. Sorting transformation: $n=-1 / 2$. The far-field spot is tilted by an angle $\theta(B)=B b^{2} /(k a)$ with respect to the optical axis, and forms an angle with the $x$ axis equal to $-2 \vartheta_{0}$. An astigmatic phase term is induced by a focal difference $\Delta f$, over a focal length $f$, between two orthogonal axes rotated by $\vartheta_{0}$ with respect to the $x$-axis positive direction. The corresponding phase term is given by

$$
\phi=\frac{\pi \Delta f}{2 \lambda f^{2}} r^{2} \cos \left[2\left(\vartheta-\vartheta_{0}\right)\right] .
$$

Then, the transferred phase exhibits a multipole-phase structure as in Eq. (D1), with $m=+2$, and strength $B=$ $\pi \Delta f /\left(2 \lambda f^{2}\right)$.

Input internal dipole: $\boldsymbol{m}=\mathbf{- 1}$. Sorting transformation: $n=+1$. The far-field spot is tilted by an angle
$\theta(B)=B b^{-1} /(k a)$, and forms an angle with the $x$ axis, which is equal to $\vartheta_{0}$. If we consider the magnetic potential generated by a magnetic moment $\mu$ on the $x-y$ plane:

$$
\mathbf{A}=\frac{\mu_{0}}{4 \pi} \frac{\boldsymbol{\mu} \times \mathbf{r}}{r^{2}} .
$$

By integrating in the $z$ direction, the imparted phase is equal to

$$
\phi=\frac{q}{\hbar} \int_{-\infty}^{+\infty} A_{z} d z=N \frac{q \mu_{0} \mu_{B}}{4 \hbar} \frac{\cos \left(\vartheta-\vartheta_{0}\right)}{r}
$$

where we assume the Bohr magneton as the unit for the magnetic moment, i.e., $|\boldsymbol{\mu}|=N \mu_{B}$. Then, the transferred phase exhibits a multipole-phase structure as in Eq. (D1), with $m=-1$, and strength $B=N q \mu_{0} \mu_{B} /(4 \hbar)$. For the simulations in Fig. 3, a 500× magnification is considered, which is a practical experimental value in electron microscopes.

\section{APPENDIX E: SORTING OF MULTIPOLE PHASE PROFILES WITH A CASCADE OF TWO CIRCULAR-SECTOR TRANSFORMATIONS}

We consider the initial phase profile:

$$
\Phi^{\text {in }}(r, \vartheta)=B r^{m} \cos \left[m\left(\vartheta-\vartheta_{0}\right)\right] .
$$

If we apply a circular-sector transformation of a factor $n_{1}$, and parameters $\left(a_{1}, b_{1}\right)$

$$
\left(\rho^{\prime}, \varphi^{\prime}\right)=\left[a_{1}\left(\frac{r}{b_{1}}\right)^{-\left(1 / n_{1}\right)}, \frac{\vartheta}{n_{1}}\right],
$$
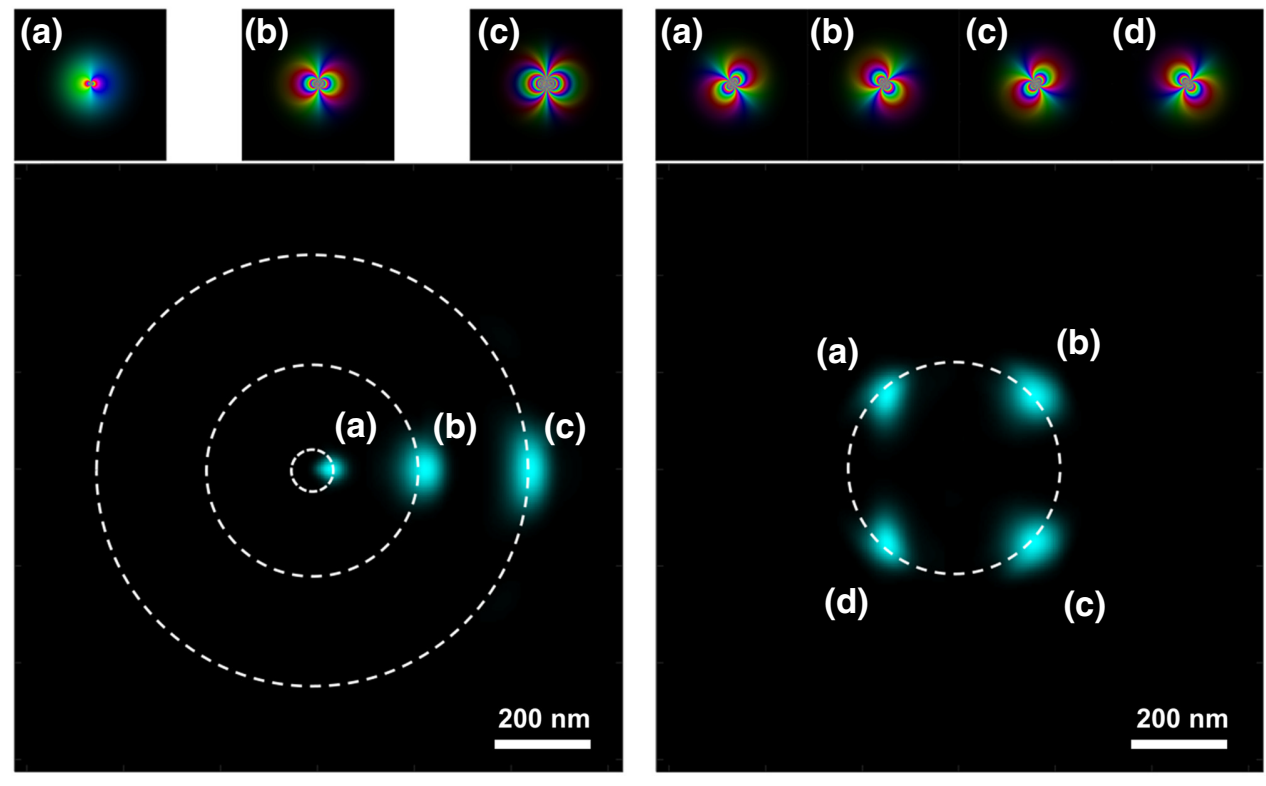

FIG. 4. Numerical simulations of magnetic dipole sorting using a sequence of two circular-sector transformations with parameters $\left(n_{1}, n_{2}\right)=(-2,+1 / 2)$. The input dipole phase is transformed into a far-field spot with position depending on the dipole strength [left panel, $1 \times 10^{6} \mu_{B}$ (a), $5 \times$ $10^{6} \mu_{B}$ (b), $10 \times 10^{6} \mu_{B}$ (c)], and rotation (right panel, $5 \times 10^{6} \mu_{B}$ ). In each subfigure, the inset plots show the input dipole field, where brightness and colors refer to intensity and phase, respectively. 
followed by a second circular-sector transformation by a factor $n_{2}$, and parameters $\left(a_{2}, b_{2}\right)$

$$
(\rho, \varphi)=\left[a_{2}\left(\frac{\rho^{\prime}}{b_{2}}\right)^{-\left(1 / n_{2}\right)}, \frac{\varphi^{\prime}}{n_{2}}\right],
$$

we obtain the new kind of transformation:

$$
(\rho, \varphi)=\left[\frac{a_{2} a_{1}^{-1 / n_{2}}}{b_{2}^{-1 / n_{2}}}\left(\frac{r}{b_{1}}\right)^{1 / n_{1} n_{2}}, \frac{\vartheta}{n_{1} n_{2}}\right],
$$

in which the exponent changes sign with respect to each separate transformation. This phase change makes this mapping holomorphic, as opposed to each of the separate mappings, which are antiholomorphic. This extends claim $l$ to every possible conformal transformation.

The final phase term is given by

$$
\Phi^{\text {out }}(\rho, \varphi)=B^{\text {out }} \rho^{m n_{1} n_{2}} \cos \left(m n_{1} n_{2} \varphi-m \vartheta_{0}\right),
$$

where $B^{\text {out }}=B b_{1}^{m} b_{2}^{-m n_{1}} /\left(a_{1}^{-m n_{1}} a_{2}^{m n_{1} n_{2}}\right)$. With the choice $n_{1} n_{2}=+1 / m$, and considering an input dipole-phase function, i.e., $m=-1$, Eq. (E5) reduces to the plane-wave term

$$
\Phi^{\text {out }}(\rho, \varphi)=B^{\text {out }} \rho \cos \left(\varphi+\vartheta_{0}\right),
$$

where $B^{\text {out }}=B b_{1}^{-1} b_{2}^{n_{1}} /\left(a_{1}^{n_{1}} a_{2}\right)$.

Therefore, the dipole-phase sorting can be performed by cascading two distinct CSTs, with factors $n_{1}$ and $n_{2}$ satisfying the condition $n_{1} n_{2}=-1$. In Fig. 4, the transformation of an input dipole-phase profile into an output spot is shown, by applying in sequence a set of transformations with factors $\left(n_{1}, n_{2}\right)=(-2,+1 / 2)$. From a practical point of view, although each transformation requires two separated elements, i.e., transformer and phase corrector, the first phase corrector and the second transformer can be integrated into a single element, therefore reducing to three the total number of phase plates to be realized.

[1] F. A. Muller, The equivalence myth of quantum mechanics; part I, Stud. Hist. Phil. Mod. Phys. 28, 35 (1997).

[2] F. A. Muller, The equivalence myth of quantum mechanics; part II, Stud. Hist. Phil. Mod. Phys. 28, 219 (1997).

[3] W. H. Zurek, Decoherence, einselection, and the quantum origins of the classical, Rev. Mod. Phys. 75, 715 (2003).

[4] M. J. Padgett, Orbital angular momentum 25 years on, Opt. Express 25, 11265 (2017).

[5] J. Harris, V. Grillo, E. Mafakheri, G. C. Gazzadi, S. Frabboni, R. W. Boyd, and E. Karimi, Structured quantum waves, Nat. Phys. 11, 629 (2015).

[6] K. Y. Bliokh, Y. P. Bliokh, S. Savel'ev, and F. Nori, Semiclassical Dynamics of Electron Wave Packet States with Phase Vortices, Phys. Rev. Lett. 99, 190404 (2007).
[7] J. Verbeeck, H. Tian, and P. Schattschneider, Production and application of electron vortex beams, Nature 467, 301 (2010).

[8] B. J. McMoran, A. Agrawal, I. M. Anderson, A. A. Herzing, H. J. Lezec, J. J. McClelland, and J. Unguris, Electron vortex beams with high quanta of orbital angular momentum, Science 331, 192 (2011).

[9] G. C. G. Berkhout, M. P. J. Lavery, J. Courtial, M. W. Beijersbergen, and M. J. Padgett, Efficient Sorting of Orbital Angular Momentum States of Light, Phys. Rev. Lett. 105, 153601 (2010).

[10] M. P. J. Lavery, D. J. Robertson, G. C. G. Berkhout, G. D. Love, M. J. Padgett, and J. Courtial, Refractive elements for the measurements of the orbital angular momentum of a single photon, Opt. Express 20, 2110 (2012).

[11] R. Fickler, R. Lapkiewicz, N. Huber, M. P. J. Lavery, M. J. Padgett, and A. Zeilinger, Interface between path and orbital angular momentum entanglement for highdimensional photonic quantum information, Nat. Commun. 5, 4502 (2014).

[12] G. Ruffato, M. Massari, G. Parisi, and F. Romanato, Test of mode-division multiplexing and demultiplexing in free-space with diffractive transformation optics, Opt. Express 25, 7859 (2017).

[13] G. Ruffato, M. Girardi, M. Massari, E. Mafakheri, B. Sephton, P. Capaldo, A. Forbes, and F. Romanato, A compact diffractive sorter for high-resolution demultiplexing of orbital angular momentum beams, Sci. Rep. 8, 10248 (2018).

[14] B. Sephton, A. Dudley, G. Ruffato, F. Romanato, L. Marrucci, M. Padgett, S. Goyal, F. Roux, T. Konrad, and A. Forbes, A versatile quantum walk resonator with bright classical light, PLoS One 14, e0214891 (2019).

[15] V. Grillo, A. H. Tavabi, F. Venturi, H. Larocque, R. Balboni, G. C. Gazzadi, S. Frabboni, P.-H. Lu, E. Mafakheri, F. Bouchard, R. E. Dunin-Borkowski, R. W. Boyd, M. P. J. Lavery, M. J. Padgett, and E. Karimi, Measuring the orbital angular momentum spectrum of an electron beam, Nat. Commun. 8, 15536 (2017).

[16] B. J. McMorran, T. R. Harvey, and M. P. J. Lavery, Efficient sorting of free electron orbital angular momentum, New J. Phys. 19, 023053 (2017).

[17] G. Pozzi, V. Grillo, P.-H. Lu, A. H. Tavabi, E. karimi, and R. E. Dunin-Borkowski, Design of electrostatic phase elements for sorting the orbital angular momentum of electrons, Ultramicroscopy 208, 112861 (2020).

[18] A. H. Tavabi, P. Rosi, E. Rotunno, A. Roncaglia, L. Belsito, S. Frabboni, G. Pozzi, G. C. Gazzadi, P.-H. Lu, R. Nijland, M. Ghosh, P. Tiemeijer, E. Karimi, R. E. Dunin-Borkowski, and V. Grillo, Experimental Demonstration of an Electrostatic Orbital Angular Momentum Sorter for Electron Beams, Phys. Rev. Lett. 126, 094802 (2021).

[19] W. J. Hossack, A. M. Darling, and A. Dahdouh, Coordinate transformations with multiple computer-generated optical elements, J. Mod. Optic. 34, 1235 (1987).

[20] M. Born and E. Wolf, Principles of Optics (Pergamon Press, New York, 1980).

[21] The curl should be intended on the three-dimensional vector field $(u, v, 0)$. In particular, we consider the $z$ component, that is $\partial_{x} v-\partial_{y} u=0$. 
[22] M. Beleggia, T. Kasama, R. E. Dunin-Borkowski, S. Hofmann, and G. Pozzi, Direct measurement of the charge distribution along a biased carbon nanotube bundle using electron holography, Appl. Phys. Lett. 98, 243101 (2011).

[23] G. Ruffato, M. Massari, and F. Romanato, Multiplication and division of the orbital angular momentum of light with diffractive transformation optics, Light Sci. Appl. 8, 113 (2019).

[24] S. Takashima, H. Kobayashi, and K. Iwashita, Integer multiplier for the orbital angular momentum of light using a circular-sector transformation, Phys. Rev. A 100, 063822 (2019).

[25] N. Engheta, On fractional calculus and fractional multipoles in electromagnetism, IEEE T. Antenn. Propag. 44, 554 (1996).

[26] N. Engheta, Electrostatic "fractional" image methods for perfectly conducting wedges and cones, IEEE T. Antenn. Propag. 44, 1565 (1996).

[27] G. Matteucci, G. F. Missiroli, M. Muccini, and G. Pozzi, Electron holography in the study of the electrostatic fields: The case of charged microtips, Ultramicroscopy 45, 77 (1992).

[28] D. J. Griffiths and Y. Li, Charge density on a conducting needle, Am. J. Phys. 64, 706 (1996).

[29] J. B. Ross, Plotting the charge distribution of a close-loop conducting wire using a microcomputer, Am. J. Phys. 55, 948 (1987).
[30] A. H. Tavabi, M. Beleggia, V. Migunov, A. Savenko, O. Öktem, R. E. Dunin-Borkowski, and G. Pozzi, Tunable ampere phase plate for low dose imaging of biomolecular complexes, Sci. Rep. 8, 1 (2018).

[31] S. Scholes, V. Rodriguez-Fajardo, and A. Forbes, Lossless reshaping of structured light, J. Opt. Soc. Am. A 37, C80 (2020).

[32] G. Ruffato, P. Capaldo, M. Massari, E. Mafakheri, and F. Romanato, Total angular momentum sorting in the telecom infrared with silicon pancharatnamberry transformation optics, Opt. Express 27, 15750 (2019).

[33] J. Verbeeck, A. Béché, K. Muller-Caspary, G. Guzzinati, M. A. Luong, and M. Den Hertog, Demonstration of a $2 \times 2$ programmable phase plate for electrons, Ultramicroscopy 190, 58 (2018).

[34] F. Zemlin, K. Weiss, P. Schiske, W. Kunath, and K. H. Herrmann, Coma free alignment of high resolution electron microscopy with the aid of optical diffractograms, Ultramicroscopy 3, 48 (1978).

[35] S. Sievers, K-F Braun, D. Eberbeck, S. Gustafsson, E. Olsson, H. W. Schumacher, and U. Siegner, Quantitative measurement of the magnetic moment of individual magnetic nanoparticles by magnetic force microscopy, Small $\mathbf{8}$, 2675 (2012).

[36] J. D. Jackson, Classical Electrodynamics, 2nd edn (John Wiley \& Sons, Inc., New York, 1975). 